\title{
Elevated oxidative stress in the aortic media of patients with bicuspid aortic valve
}

\author{
Marie Billaud, PhD, ${ }^{\mathrm{a}, \mathrm{c}, \mathrm{d}}$ Julie A. Phillippi, PhD, ${ }^{\mathrm{a}, \mathrm{b}, \mathrm{c}, \mathrm{d}}$ Mary P. Kotlarczyk, PhD, ${ }^{\mathrm{a}, \mathrm{d}}$ Jennifer C. Hill, MFS, \\ Bradley W. Ellis, BS, ${ }^{b}$ Claudette M. St Croix, PhD, ${ }^{\mathrm{e}}$ Nadiezhda Cantu-Medéllin, BS, ${ }^{\mathrm{f}}$ Eric E. Kelley, PhD, ${ }^{\mathrm{g}}$ \\ and Thomas G. Gleason, MD ${ }^{\mathrm{a}, \mathrm{b}, \mathrm{c}, \mathrm{d}}$
}

\section{ABSTRACT}

Objective: Congenital bicuspid aortic valve (BAV) is distinctly associated with the development of ascending aortopathy in adulthood, portending risk of both ascending aortic aneurysm and dissection. Our previous work implicated deficiency in oxidative stress response as a mediator of the BAV-associated aortopathy. We hypothesize that reactive oxygen species generation invokes elevated local oxidative tissue damage in ascending aorta of patients with BAV.

Methods: Ascending aortic specimens were obtained from patients undergoing elective aortic replacement and/or aortic valve replacement and during heart transplant operations. Levels of superoxide anion were measured via highpressure liquid chromatography-based detection of 2-hydroxyethidium in aortic specimens. Lipid peroxidation and enzymatic activity of superoxide dismutase and peroxidase were quantified in aortic specimens.

Results: Superoxide anion production was elevated in aortic specimens from patients with nonaneurysmal BAV $(\mathrm{n}=59)$ compared with specimens from patients with the morphologically normal tricuspid aortic valve (TAV, $\mathrm{n}=38$ ). Total superoxide dismutase activity was similar among aortic specimens from patients with TAV versus BAV ( $\mathrm{n}=27$ and 26 , respectively), whereas peroxidase activity was increased in aortic specimens from patients with BAV compared with specimens from patients with TAV ( $\mathrm{n}=14$ for both groups). Lipid peroxidation was elevated in aortic specimens from BAV patients compared with TAV patients $(n=14$ and 11 , respectively).

Conclusions: Superoxide anion accumulation and increased lipid peroxidation demonstrate that, despite increased peroxidase activity, the ascending aortopathy of patients with BAV involves oxidative stress. In addition, the absence of increased superoxide dismutase activity in BAV specimens indicates a deficiency in antioxidant defense. This suggests that the characteristic smooth muscle cell loss observed in BAV aortopathy may be a consequence of superoxidemediated cell damage. (J Thorac Cardiovasc Surg 2017;154:1756-62)

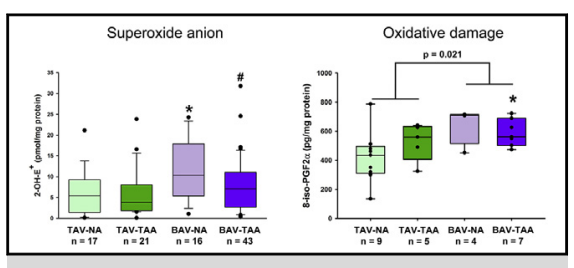

Increased superoxide anion and oxidative damage in the proximal aorta of patients with bicuspid aortic valve.

\section{Central Message}

Levels of superoxide anion and oxidative damages are elevated in the ascending aorta of patients with bicuspid aortic valve.

\section{Perspective}

In patients with bicuspid aortic valve (BAV), the proximal aorta is characterized by heightened superoxide anion production and oxidative damage. This oxidative environment may adversely impact cellular and matrix homeostasis and could explain the unique manifestation of BAV aortopathy. Identifying the impact of oxidative stress on local biological parameters may offer a novel perspective for the management of BAV aortopathy.

See Editorial Commentaries pages 1763 and 1764.

\footnotetext{
From the Departments of ${ }^{\mathrm{a}}$ Cardiothoracic Surgery, ${ }^{\mathrm{b}}$ Bioengineering, ${ }^{\mathrm{e}}$ Environmental and Occupational Health, and ${ }^{\mathrm{f}}$ Anesthesiology, ${ }^{\mathrm{c}} \mathrm{McGowan}$ Institute for Regenerative Medicine, and ${ }^{\mathrm{d} C e n t e r}$ for Vascular Remodeling and Regeneration, University of Pittsburgh, Pittsburgh, Pa; and ${ }^{\mathrm{g}}$ Department of Physiology and Pharmacology, West Virginia University, Morgantown, WVa.

M.B. and J.A.P. contributed equally to this article.

Research reported in this publication was supported by the National Heart, Lung and Blood Institute of the National Institutes of Health, grant HL109132 (TGG).

Received for publication Oct 7, 2016; revisions received May 3, 2017; accepted for publication May 18, 2017; available ahead of print June 23, 2017.

Address for reprints: Thomas G. Gleason, MD, Ronald V. Pellegrini Professor and Chief, Division of Cardiac Surgery, Department of Cardiothoracic Surgery, University of Pittsburgh School of Medicine, 5200 Centre Ave, Suite 715, Pittsburgh, PA 15232 (E-mail: gleasontg@upmc.edu). $0022-5223 / \$ 36.00$

Copyright @ 2017 Published by Elsevier Inc. on behalf of The American Association for Thoracic Surgery

http://dx.doi.org/10.1016/j.jtcvs.2017.05.065
}

Bicuspid aortic valve (BAV) is the most prevalent congenital heart malformation ${ }^{1}$ and is associated with an increased risk of developing thoracic aortic aneurysm (TAA) or dissection in the ascending thoracic aorta. ${ }^{2}$ Risk stratification for aortic catastrophe among patients with BAV is challenging because the cause of BAV formation and the

Scanning this QR code will take you to the article title page. 

Abbreviations and Acronyms
$\begin{array}{ll}\text { BAV } & =\text { bicuspid aortic valve } \\ \text { DHE } & =\text { dihydroethidium }\end{array}$
$\mathrm{ECM}=$ extracellular matrix
eNOS = endothelial nitric oxide synthase
NOX $=$ NADPH oxidase
$\mathrm{O}_{2}{ }^{--} \quad=$ superoxide anion
2-OH-E $+=2$-hydroxyethidium
PBS $=$ phosphate-buffered saline
ROS $=$ reactive oxygen species
SMC $=$ smooth muscle cells
SOD $=$ superoxide dismutase
TAA $=$ thoracic aortic aneurysm
TAV $=$ tricuspid aortic valve

upstream effector molecules and consequent mediating pathways of the associated aortopathy are not understood fully.

Oxidative stress has been implicated in idiopathic TAA, ${ }^{3}$ Marfan syndrome, ${ }^{4}$ and abdominal aortic aneurysms. ${ }^{5}$ However, the young age-related incidence of TAA formation in the patient with BAV, asymmetric dilation pattern localized to the proximal ascending aorta, the lack of multiorgan involvement, and the noninflammatory nature of the pathology indicate that the pathophysiology of BAVassociated aortopathy is distinct from that of the abdominal aorta, idiopathic TAAs, and TAAs arising in patients with connective tissue disorders. Oxidative stress response has been proposed by our group as a mediator of BAVassociated aortopathy from observations of increased susceptibility to oxidative stress-induced cell death of primary medial smooth muscle cells (SMCs) from patients with BAV compared with age-matched controls with normalcaliber aorta. ${ }^{6}$ These findings led us to hypothesize that reactive oxygen species (ROS) generation invokes elevated local oxidative tissue damage in ascending aortic specimens of patients with BAV.

In the present study, we sought to investigate superoxide anion $\left(\mathrm{O}_{2}{ }^{\bullet-}\right)$ and lipid peroxidation levels in ascending aortic specimens of patients with BAV ex vivo. We also examined superoxide dismutase (SOD) activity, a major ROS defense mechanism, as well as peroxidase activity in BAV specimens. Collectively, our results suggest that an environment of heightened oxidative stress and consequent oxidative damage to the ascending aorta contribute to BAVassociated aortopathy.

\section{METHODS}

\section{Tissue Collection}

Human ascending aortic specimens $(n=130)$ were collected, with approval of the institutional review board (\#PRO07020120 - approved on February 26, 2015) and informed patient consent, from patients undergoing elective aortic replacement due to aneurysm or aortic valve replacement in the absence of an aneurysm. All procedures performed were in accordance with institutional guidelines. Nonaneurysmal ascending aortic specimens were collected from local heart transplant donor and recipient patients with tricuspid aortic valve (TAV) and no history of aortic disease or aortic valve disease, with approval of the institutional research board and informed patient consent or approval from the Center for Organ Recovery and Education. Patient demographics, age, sex, aortic diameter, degree of aortic insufficiency and/or stenosis, valve morphology (tricuspid or bicuspid) and relevant comorbidities (eg, hypertension and smoking) were recorded carefully and are displayed in Table 1. Ascending aortic specimens were categorized as aneurysmal when the maximal orthogonal aortic diameter exceeded $42 \mathrm{~mm}$. Patients clinically diagnosed with a known connective tissue disorder, such as Marfan, Ehlers-Danlos, and Loeys-Dietz syndrome, were excluded from this study.

BAV morphotype was assessed intraoperatively in almost all cases, according to the Sievers classification. ${ }^{7}$ Among the 22 patients with nonaneurysmal BAV, 17 (77\%) presented with a type $1 \mathrm{BAV}(15$ type $1 \mathrm{~L} / \mathrm{R}, 1$ type $1 \mathrm{R} /$ $\mathrm{N}, 1$ type $1 \mathrm{~L} / \mathrm{N})$ and 1 patient $(4.5 \%)$ exhibited a type $2 \mathrm{BAV}$ morphotype. The majority $(60 \%)$ of the 53 patients with aneurysmal BAV presented with a type $1 \mathrm{BAV}$ (including 26 type $1 \mathrm{~L} / \mathrm{R}, 5$ type $1 \mathrm{R} / \mathrm{N}$, and 1 type $1 \mathrm{~L} / \mathrm{N}$ ) whereas 4 patients $(7.5 \%)$ exhibited a type $0 \mathrm{BAV}$. The frequency of BAV morphotypes is consistent with previous work from our laboratory ${ }^{8}$ and others. ${ }^{7,9}$

On excision, aortic specimens were placed in cold saline and transported to the laboratory, where samples of smaller size were sectioned for various assay. An effort was made to maintain the time elapsed between specimen excision and harvesting constant. Samples from similar regions of the aorta were used for each assay to adequately compare the data. The adventitial layer was stripped carefully from the tunica media and the intima was gently scraped away. Medial specimens were processed as described herein for various assays.

High-Pressure Liquid Chromatography (HPLC)Based Detection of 2-Hydroxyethidium (2-OH-E + )

$2-\mathrm{OH}-\mathrm{E}^{+}$has been shown to be the lone reaction product of $\mathrm{O}_{2}{ }^{\bullet-}$ with dihydroethidium (DHE), making its detection via HPLC the most sensitive

TABLE 1. Demographics for all patients enrolled in the study

\begin{tabular}{|c|c|c|c|c|}
\hline & \multicolumn{2}{|c|}{ TAV } & \multicolumn{2}{|c|}{ BAV } \\
\hline & NA & TAA & NA & TAA \\
\hline $\mathrm{n}, \mathrm{M} / \mathrm{F}$ & $27(20 / 6)^{*}$ & $28(18 / 10)$ & $22(15 / 7)$ & $53(43 / 10)$ \\
\hline Age, $y$ & $59.5 \pm 16$ & $64.0 \pm 10$ & $56 \pm 14 \dagger$ & $56 \pm 11 \dagger$ \\
\hline Diameter, mm & $\leq 42$ & $52.0 \pm 5.2$ & $38 \pm 4.28 \dagger, \ddagger$ & $49.0 \pm 4.3 \dagger, \S$ \\
\hline Aortic index & $\leq 2.20$ & $2.60 \pm 0.52$ & $1.82 \pm 0.27 \dagger$ & $2.34 \pm 0.38 \dagger, \S$ \\
\hline HTN & $67 \%$ & $79 \%$ & $68 \%$ & $71 \%$ \\
\hline Smoking & $83 \%$ & $67 \%$ & $57 \%$ & $55 \%$ \\
\hline \multicolumn{5}{|l|}{ AI } \\
\hline $1+$ & $6 \%$ & $26 \%$ & $29 \%$ & $26 \%$ \\
\hline $2-3+$ & $25 \%$ & $30 \%$ & $10 \%$ & $14 \%$ \\
\hline $4+$ & $6 \%$ & $15 \%$ & $19 \%$ & $12 \%$ \\
\hline \multicolumn{5}{|l|}{ AS } \\
\hline Mild & $0 \%$ & $0 \%$ & $0 \%$ & $8 \%$ \\
\hline Mod & $0 \%$ & $0 \%$ & $5 \%$ & $16 \%$ \\
\hline Severe & $15 \%$ & $9 \%$ & $71 \% \dagger, \|$ & $49 \% \dagger, \|$ \\
\hline
\end{tabular}

Data are expressed as median \pm SD (age and diameter), or as percentage (HTN, smoking, AI, and AS). TAV, Tricuspid aortic valve; $B A V$, bicuspid aortic valve; $N A$, nonaneurysmal; $T A A$, thoracic aortic aneurysm; $n$, number of patients enrolled; $M$, male; $F$, female; $H T N$, hypertension; $A I$, aortic insufficiency; $A S$, aortic stenosis; Mod, moderate. *The sex of 1 patient was unknown. $\dagger, \S$, $\|$ Indicate $P<.05$ versus TAV-TAA, BAV-NA, and TAV-NA, respectively, as assessed by $\chi^{2}$ test or a MannWhitney $U$ test. $\ddagger$ The diameter of 7 patients was unknown. 
and accurate method to determine $\mathrm{O}_{2}{ }^{\bullet-}$ generation in biological specimens. ${ }^{10}$ A portion $\left(35-40 \mathrm{mg}, 1-2 \mathrm{~cm}^{2}\right)$ of fresh aortic media was incubated in $10 \mu \mathrm{mol} / \mathrm{L}$ DHE (Sigma-Aldrich, St Louis, Mo) in phosphate-buffered saline (PBS; Life Technologies, Carlsbad, Calif) for 30 minutes in the dark at $37^{\circ} \mathrm{C}$ with $5 \% \mathrm{CO}_{2}$ and humidity. The DHE-labeled tissue was then snap-frozen in liquid nitrogen and homogenized with the gentleMACS Dissociator (Miltenyi Biotec Inc, San Diego, Calif). The total homogenate was further passed through a 28.5-gauge needle in $150 \mu \mathrm{L} 0.1 \%$ Triton X100 in PBS. Twenty microliters of the homogenate was reserved for total protein determination with the BCA Protein Assay Kit (Pierce, Rockford, IIl). One hundred microliters of the homogenate was diluted 1:1 in extraction buffer $(0.2 \mathrm{~mol} / \mathrm{L}$ perchloric acid in methanol $)$ and incubated on ice for 2 hours. The samples were centrifuged at $20,000 \mathrm{~g}$ for 30 minutes at $4^{\circ} \mathrm{C}$. Samples $(120 \mu \mathrm{L})$ were then diluted $1: 1$ in $1 \mathrm{M}$ phosphate buffer $(0.7 \mathrm{~mol} / \mathrm{L}$ potassium phosphate monobasic and $0.3 \mathrm{~mol} / \mathrm{L}$ phosphoric acid, $\mathrm{pH} 2.6$ ), followed by centrifugation at 20,000g for 15 minutes at $4{ }^{\circ} \mathrm{C}$. The supernatant $(200 \mu \mathrm{L})$ was subjected to HPLC analysis for electrochemical detection (ESA CoulArray 5600) of 2-OH-E ${ }^{+}$. An ether-linked phenyl column $(100 \mathrm{~mm} \times 4.6 \mathrm{~mm})$ was used with 2 mobile phases (Solution A: $50 \mathrm{mmol} / \mathrm{L}$ phosphate buffer in $90 \%$ water and $10 \%$ acetonitrile; Solution B: $50 \mathrm{mmol} / \mathrm{L}$ phosphate buffer in $40 \%$ water and $60 \%$ acetonitrile), and a gradient elution to increase the acetonitrile concentration from $25 \%$ to $60 \%$ over 10 minutes at a flow rate of $0.75 \mathrm{~mL} / \mathrm{min}$. The areas of the corresponding peaks were assessed with ESA CoulArray software (SelectScience, Bath, UK). The limit of detection for this assay was determined from a standard curve and established at $20 \mathrm{fmol}$. The concentration of $2-\mathrm{OH}-\mathrm{E}^{+}$in each sample was calculated with a standard curve and normalized to the protein concentration of the tissue lysate. Specimens with undetectable $2-\mathrm{OH}-\mathrm{E}^{+}$were assigned values at the limit of detection $(20 \mathrm{fmol})$ to enable statistical analyses on all specimens interrogated. As such, 11 of a total of 97 samples were assigned this limit of detection.

\section{Quantification of SOD and Peroxidase Activity}

For determination of antioxidant activities, snap-frozen aortic media tissue samples were homogenized in cold lysis buffer $(20 \mathrm{mmol} / \mathrm{L}$ HEPES buffer solution, pH 7.2, $1 \mathrm{mmol} / \mathrm{L}$ ethylene glycol-bis $(\beta$-aminoethyl ether)-N,N, $\mathrm{N}^{\prime}, \mathrm{N}^{\prime}$-tetraacetic acid, $210 \mathrm{mmol} / \mathrm{L}$ mannitol, and $70 \mathrm{mmol} / \mathrm{L}$ sucrose; all Sigma-Aldrich) for SOD activity and in PBS $\left(\mathrm{Ca}^{2+} \mathrm{Mg}^{2+}\right.$-free $)$ for the peroxidase assay with the gentleMACS Dissociator (Miltenyi Biotec Inc). Total SOD activity was determined according to the manufacturer's instructions (Cayman Chemical, Ann Arbor, Mich). Peroxidase activity was quantified by a modification of the method of Weydert and Cullen. ${ }^{11}$ To summarize, homogenized medial specimens were diluted 1:1 in PBS and absorbance was measured at $240 \mathrm{~nm}$ every 8 seconds for 24 seconds after the addition of $30 \mathrm{mmol} / \mathrm{L} \mathrm{H}_{2} \mathrm{O}_{2}$. Peroxidase activity $(\mathrm{K})$ was calculated as previously described. ${ }^{11}$ SOD and peroxidase activity levels were normalized to total protein content as determined by the Bradford method (Bio-Rad, Hercules, Calif).

\section{Detection of Lipid Peroxidation}

To assess the oxidative damage at the tissue level, lipid peroxidation was assessed by quantification of 8 -iso-prostaglandin $\mathrm{F} 2 \alpha$, resulting from the nonenzymatic peroxidation of arachidonic acid in membrane phospholipids. ${ }^{12}$ Aliquots of aortic tissue lysates were prepared as described previously for the SOD and peroxidase activity assays and used to determined amount of lipid peroxidation using the Oxiselect 8-iso-prostaglandin F2 $\alpha$ ELISA kit (Cell BioLabs Inc, San Diego, Calif) according to the manufacturer's instructions. The amount of 8-iso-prostogladin F2 $\alpha$ was measured from aortic tissue lysate against a standard curve. Results were further normalized to the protein concentration and expressed as $\mathrm{pg} / \mathrm{mg}$ protein of tissue lysate.

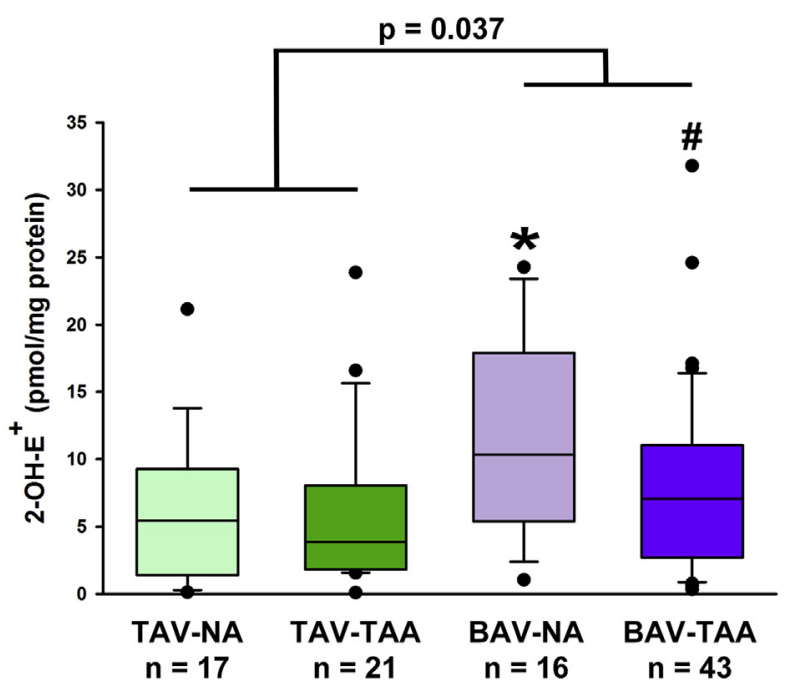

FIGURE 1. Detection of $\mathrm{O}^{\bullet-}$ generation. HPLC-based detection of 2-OH-E ${ }^{+}$in aortic media showing a significant increase in $\mathrm{O}_{2}{ }^{--}$in the ascending aorta of patients with BAV. Box plots depict the median (middle line of the box), first (lower line of the box), and third (upper line of the box) quartiles with errors bars representing 90th and 10th percentiles. Data points distributed below the 10th percentile and above the 90th percentile are indicated by a closed circle. *Indicates $P<.05$ from TAVNA and \#Indicates $P<.05$ from BAV-NA using a Kruskal-Wallis test. $P=.037$ indicates a statistically significant difference between all BAV specimens and all TAV specimens using a Mann-Whitney $U$ nonparametric test. 2-OH-E $E^{+}$2-hydroxyethidium; TAV-NA, tricuspid aortic valvenonaneurysmal; TAV-TAA, tricuspid aortic valve-thoracic aortic aneurysm; $B A V-N A$, bicuspid aortic valve-nonaneurysmal; $B A V-T A A$, bicuspid aortic valve-thoracic aortic aneurysm.

\section{Statistical Analyses}

All results mentioned in the text are expressed as mean \pm standard error of the mean and are represented in figures as median and interquartile range with errors bars representing 90th and 10th percentiles. Data in Table 1 are expressed as median \pm standard deviation. Experimental outliers were identified and excluded from analysis with the Outlier Labeling Rule according to Hoaglin and Iglewicz ${ }^{13}$ and Tukey. Statistical tests were performed using SPSS, version 22 (IBM Corp, Armonk, NY). Comparisons between group demographics were assessed by $\chi^{2}$ test or a MannWhitney $U$ test. All experimental endpoints were compared in SPSS for the 4 patient cohorts with the nonparametric Kruskal-Wallis test. When experimental endpoints were compared between TAV versus BAV specimens, the Mann-Whitney $U$ nonparametric test was performed. A $P$ value of less than .05 was considered statistically significant.

\section{RESULTS}

\section{Levels of $\mathrm{O}_{2}{ }^{--}$Are Increased in the Aortic Media of BAV Specimens}

Quantification of 2-OH-E ${ }^{+}$, the specific oxidation product of DHE by $\mathrm{O}_{2}{ }^{--}$, showed that aortic specimens from nonaneurysmal $\mathrm{BAV}$ have the highest level of $\mathrm{O}_{2}{ }^{\bullet-}$ (Figure 1, $11.7 \pm 1.86 \mathrm{pmol} / \mathrm{pg}$ protein). The level of $\mathrm{O}_{2}{ }^{--}$in nonaneurysmal BAV specimens was increased compared with $\mathrm{O}_{2}{ }^{\bullet-}$ levels from nonaneurysmal TAV and aneurysmal BAV aortic specimens (Figure 1, $6.41 \pm 1.30$ 
$\mathrm{pmol} / \mathrm{mg}, P=.041$ and $7.80 \pm 1.02 \mathrm{pmol} / \mathrm{mg}, P=.042$, respectively).

\section{Specimens From Patients With BAV Exhibit Similar SOD Activity and Increased Peroxidase Activity}

Total SOD activity was quantified in nonaneurysmal and patients with aneurysmal BAV and TAV and found to be similar among the 4 patient cohorts (Figure 2, A). Conversely, aortic specimens from patients with nonaneurysmal BAV exhibited increased peroxidase activity compared with nonaneurysmal TAV specimens (Figure 2, B, $15.9 \pm 2.29 \mathrm{~K} / \mathrm{mg}$ vs $7.18 \pm 1.14 \mathrm{~K} / \mathrm{mg}$, respectively, $P=.023)$. When combined, BAV specimens exhibited a greater peroxidase activity compared with TAV specimens independent of the presence of aneurysm (Figure 2, $B, 15.4$ $\pm 1.87 \mathrm{~K} / \mathrm{mg}$ vs $7.69 \pm 1.15 \mathrm{~K} / \mathrm{mg}$, respectively, $P<.002$ ).

\section{Lipid Peroxidation Is Increased in Aortic Specimens From Patients With BAV}

We assessed lipid peroxidation as a marker of cellular damage due to oxidative stress. Quantification of 8-iso-prostaglandin F2 $\alpha$ revealed increased lipid peroxidation in the aortic media of aneurysmal patients with BAV compared with specimens from nonaneurysmal patients with TAV (Figure $3,589 \pm 35.4 \mathrm{pg} / \mathrm{mg}$ vs $420 \pm 60.1 \mathrm{pg} / \mathrm{mg}$, respectively, $P=.023$ ). Levels of 8 -iso-prostaglandin F2 $\alpha$ were elevated in all BAV specimens compared with all TAV specimens, irrespective of the presence of aneurysm (Figure 3, $610 \pm 32.0 \mathrm{pg} / \mathrm{mg}$ vs $458 \pm 44.7 \mathrm{pg} / \mathrm{mg}, P=.021)$.

\section{DISCUSSION}

In the setting of ascending aortic aneurysm, it is accepted widely that vessel dilatation and loss of biomechanical integrity is a consequence of cystic medial degeneration, a phenomenon involving noninflammatory SMC loss and elastin fragmentation in the aortic media. ${ }^{14}$ However, aneurysms arising in the BAV population are distinct from those presenting in patients with connective tissue disorders, such as the Marfan syndrome, and from degenerative TAA occurring in patients with the morphologically normal TAV. ${ }^{15}$ Specifically, the cause of BAV remains unknown, with no single gene or set of genes identified as being responsible for the abnormal valve development and the associated aortopathy. ${ }^{16}$ From an epidemiologic standpoint, patients with BAV present for aortic replacement approximately 10 to 15 years younger than patients with TAV with degenerative aneurysms, and the frequency of TAA is estimated to be approximately $10 \%$, whereas TAA in patients with TAV occurs at a frequency of less than $0.02 \% .{ }^{17-}$ ${ }^{19}$ In addition, it is likely that all patients with BAV have a certain degree of ascending aortopathy because they do exhibit a greater ascending aortic diameter compared with TAV patients, ${ }^{19-21}$ even when matched for variables such as sex, age, or degree or valvulopathy. ${ }^{22}$ The distinct manifestations of BAV- versus TAV-associated aortopathy is further supported by our previous work at the tissue, cellular, and molecular levels showing distinct biomechanical properties associated with differences in extracellular matrix maturity and architecture ${ }^{23-27}$ and altered SMC response to oxidative stress ${ }^{6,28}$ in the BAV versus TAV aortopathy.

Despite an abundance of literature describing oxidative stress in several cardiovascular pathologies, there have been relatively few reports focusing on the involvement of ROS in thoracic aortic diseases. In this study, we have uncovered elevated $\mathrm{O}_{2}{ }^{\bullet-}$ levels along with demonstrating
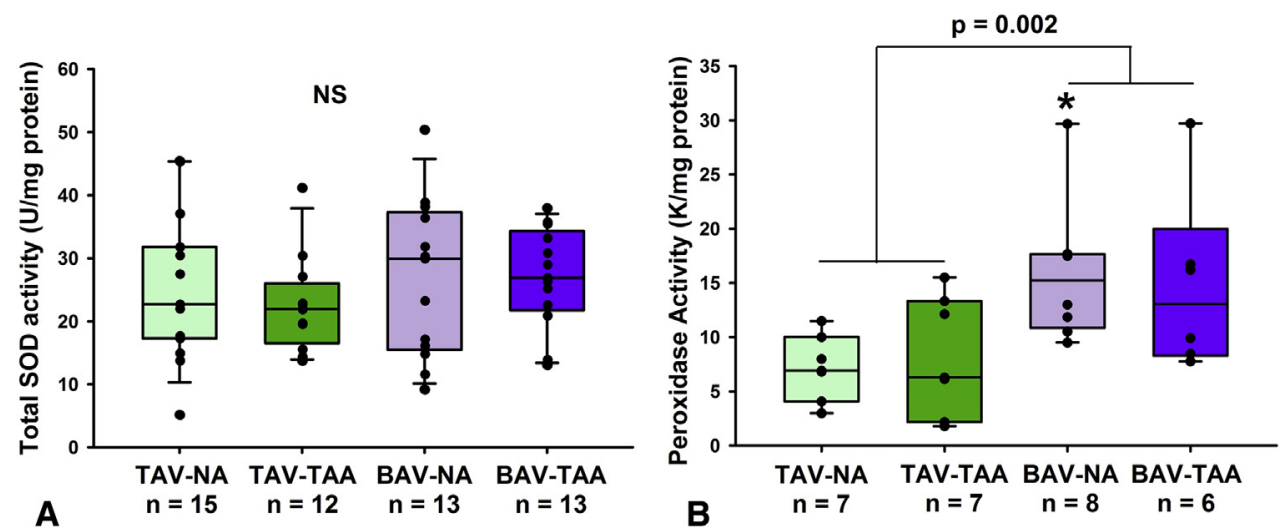

FIGURE 2. Detection of antioxidant activity in aortic media. A, Total SOD activity was similar among aortic specimens from patients with aneurysmal and nonaneurysmal BAV and TAV. B, Peroxidase activity was found to be increased in aortic specimens from patients with BAV compared with TAV specimens. Box plots depict the median (middle line of the box), first (lower line of the box), and third (upper line of the box) quartiles with errors bars representing 90th and 10th percentiles. All data points are indicated by a closed circle. *Indicates $P<.05$ from TAV-NA. In (B) $P=.002$ was obtained using a Mann-Whitney nonparametric $U$ test comparing all TAV versus all BAV specimens. SOD, Superoxide dismutase; TAV-NA, tricuspid aortic valve-nonaneurysmal; TAV-TAA, tricuspid aortic valve-thoracic aortic aneurysm; $B A V$ - $N A$, bicuspid aortic valve-nonaneurysmal; $B A V$ - $T A A$, bicuspid aortic valve-thoracic aortic aneurysm; $N S$, not significant using a Kruskal-Wallis test. 


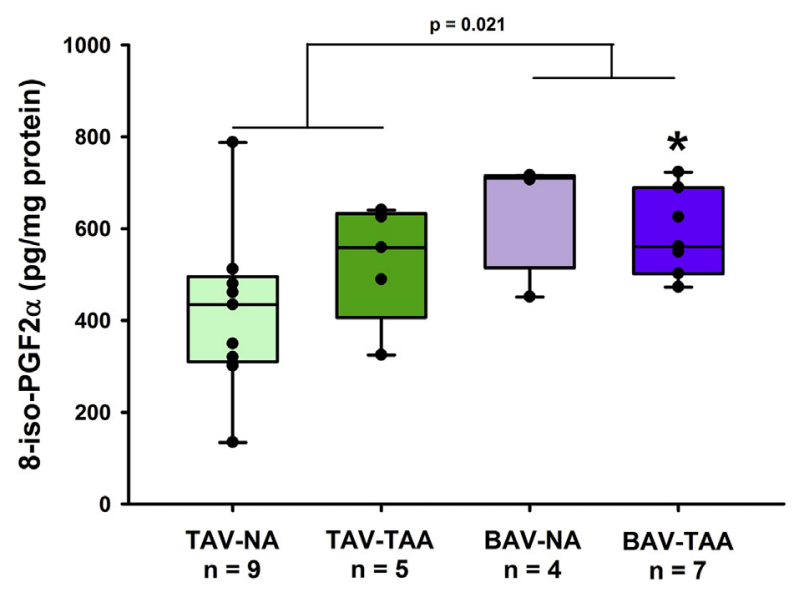

FIGURE 3. Lipid peroxidation in aortic media. Elevated oxidative damage in aortic specimens from patients with BAV was revealed by increased levels of 8 -iso-PGF $2 \alpha$ detected by ELISA. Box plots depict the median (middle line of the box), first (lower line of the box), and third (upper line of the box) quartiles with errors bars representing 90th and 10th percentiles. All data points are indicated by a closed circle. *Indicates $P<.05$ from TAV-NA via Kruskal-Wallis. A $P$ value of .021 was obtained using a Mann-Whitney nonparametric $U$ test comparing all TAV versus all BAV specimens. 8-iso-PGF2 $\alpha$, 8-iso-prostaglandin F2 $\alpha$; TAV-NA, tricuspid aortic valve-nonaneurysmal; TAV-TAA, tricuspid aortic valvethoracic aortic aneurysm; $B A V-N A$, bicuspid aortic valve-nonaneurysmal; $B A V-T A A$, bicuspid aortic valve-thoracic aortic aneurysm.

cellular oxidative damage in the thoracic aortic media of BAV aortas compared with TAV aortas. Interestingly, this increase in oxidative stress was detected in both aneurysmal and nonaneurysmal BAV specimens but was not noted in aneurysmal TAV specimens. This finding suggests that oxidative stress may play a prominent role in the onset and progression of BAV-associated aortopathy, whereas it may be less involved in the pathophysiology of degenerative aneurysms. In addition, $\mathrm{O}_{2}{ }^{\bullet-}$ levels were significantly greater in nonaneurysmal BAV specimens compared with aneurysmal BAV specimens. This may indicate that $\mathrm{O}_{2}{ }^{\bullet-}$ plays a more prominent role in the early stages of BAV aortopathy than in the later stages. Further investigation is required to determine the exact link between elevated $\mathrm{O}_{2}{ }^{--}$and the changes in extracellular matrix (ECM) composition and microarchitecture revealed by our group in specimens collected from earlier stages of BAV aortopathy. $^{24}$

Several investigators have examined the involvement of oxidative stress in animal models of TAA. For example, $\mathrm{Fbn} 1^{\mathrm{C} 1039 \mathrm{G} /+}$ mice, a mouse model of Marfan syndrome, developed TAAs ${ }^{29}$ and exhibited down-regulation of SOD expression amidst up-regulation of the expression of inducible nitric oxide synthase, NADPH oxidase (NOX) subunits, and xanthine oxidase, accompanied by increased lipid peroxidation. ${ }^{30}$ In addition, elevated levels of homocysteine and protein carbonyl contents were found in the serum of patients with Marfan syndrome along with a decrease in total antioxidant capacity. ${ }^{4}$ Although the exact role of hyperhomocysteinemia in human Marfan disease is unclear, it has been shown to cause increased deposition of collagen and degradation of elastin in the mouse aorta, ultimately leading to altered vessel biomechanics evidenced by reduced aortic flow velocity. ${ }^{31}$ In view of the paucity of studies focused on oxidative stress in patients presenting with TAA, determining whether $\mathrm{O}_{2}{ }^{--}$is a cause or a consequence of the BAV-associated aortopathy remains unknown and is warranted.

We previously uncovered a marked susceptibility to oxidative stress among SMCs from BAV-associated aortopathy specimens by demonstrating a deficiency of basal and stress-induced expression of the antioxidant metallothionein, ${ }^{6}$ a finding that was corroborated by others. ${ }^{32}$ Although peroxidase activity was found to be elevated in aortic specimens from patients with BAV, total SOD activity remained similar between patient cohorts in the present study. These results are in line with our recent study showing that the expression of the $3 \mathrm{SOD}$ isoforms is either similar (Sodl) or decreased ( $\operatorname{Sod} 2$ and $\operatorname{Sod} 3$ ) in the R region of the BAV ascending aorta, which corresponds to the aortic segment proximally adjacent to the right coronary sinus. ${ }^{8}$ In a smaller cohort of aneurysmal patients with BAV, it recently was reported that the protein level of SOD3 was decreased in the ascending aorta and associated with decreased phosphorylation level of 2 targets of SOD3, namely Erk1/2 and Akt, compared with nonaneurysmal TAV specimens. ${ }^{33}$ The same study also revealed similar levels of SOD3 expression in specimens of degenerative aneurysms and healthy aorta. ${ }^{33}$ In parallel, recent work from Branchetti and colleagues ${ }^{34}$ described down-regulation of SOD1 and SOD2 at the mRNA level in the aortic media of patient with degenerative aneurysms. Despite these recent reports focused on the expression of SODs in BAV and TAV aneurysmal aortic specimens, the enzymatic activity of SODs has not been reported previously. We found that total SOD activity was similar, suggesting an inadequate defensive response by SMCs to the increase in $\mathrm{O}_{2}{ }^{\bullet-}$ levels. The relative contributions of SOD1, SOD2, and SOD3 isoforms in overall SOD enzymatic activity in BAV aortic specimens will require further study.

Our data indicate that despite activation of the antioxidant enzyme peroxidase, we detected increased levels of 8 -iso-PGF2 $\alpha$, which are indicative of oxidative damage in BAV aortic specimens and could negatively affect cellular function. Isoprostane result from the nonenzymatic peroxidation of arachidonic acid in membrane phospholipids, thus affecting the integrity of all cell membranes. ${ }^{12,35,36}$ Lipid peroxidation was observed in aneurysmal BAV specimens despite similar levels of 2-OH-E + in these specimens. This discrepancy could be explained by the fact that other ROS such as hydrogen peroxide, hydroxyl radical, or hydroxyperoxyl radical may be involved in later stages of 
BAV aortopathy. Regardless of the nature of the ROS involved, oxidative damage in form of lipid peroxidation was detected in our aneurysmal BAV specimens. Lipid peroxidation has been associated with cardiovascular disease including atherosclerosis,${ }^{37}$ coronary artery disease, ${ }^{38}$ and aortic insufficiency. ${ }^{39}$ At the cellular level, increased lipid peroxidation is known to perturb the homeostasis of the plasma membrane, leading to cell death in several cell types including vascular SMCs. ${ }^{40}$ In the BAV aortic specimens, the high levels of 8 -iso-PGF2 $\alpha$ could thus contribute to SMC loss during cystic medial degeneration. Furthermore, mishandling of $\mathrm{O}_{2}{ }^{--}$and $\mathrm{H}_{2} \mathrm{O}_{2}$ in the BAV aorta may adversely influence SMC behavior, as shown in the study by Branchetti and colleagues, ${ }^{34}$ where treatment of human aortic SMCs with $\mathrm{H}_{2} \mathrm{O}_{2}$ resulted in alteration of cell phenotype. In addition, we previously reported that SMCs isolated from BAV aortic specimens have reduced cell viability under oxidative stress conditions when compared to SMCs from TAV aortic specimens. ${ }^{6}$ This observation was attributed to the lower levels of the antioxidant metallothionein measured in BAV aortic specimens, ${ }^{6}$ since a similar reduced cell viability under oxidative stress conditions was observed in aortic SMCs isolated from metallothionein knockout mice. ${ }^{28}$ Collectively, these studies indicate an imbalance of ROS-related mechanisms in the aortic wall of patients with BAV.

To date, the main sources of $\mathrm{O}_{2}^{\bullet-}$ production in SMCs, including NOXs and xanthine oxidase, have not been investigated in human aortic specimens. However, in a mouse model of TAA induced by angiotensin II infusion, increased expression of the NOX subunit $\mathrm{p} 22^{\text {phox }}$ was measured and found to colocalize with matrix metalloproteinase activity in the aortic media. ${ }^{41}$ More recently, we identified endothelial nitric oxide synthase (eNOS) as one probable source of $\mathrm{O}_{2}{ }^{--}$in the ascending aorta. ${ }^{42}$ Although eNOS has been implicated previously in the pathophysiology of BAVassociated aortopathy, ${ }^{42-44}$ involvement in development or progression of the associated aortopathy is mechanistically unclear. Regional down-regulation of eNOS expression has been reported in BAV aortic specimens, ${ }^{43}$ and single-nucleotide polymorphisms have been identified in patients with aneurysmal $\mathrm{BAV}^{44}$ Work from our laboratory has revealed increased eNOS expression in aortic intima-media specimens isolated from patients with $\mathrm{BAV}^{42}$ Despite this finding, nitric oxide bioavailability was not found to be concordantly elevated. Since uncoupling of eNOS has been associated with a lack of NO bioavailability and $\mathrm{O}_{2}{ }^{\bullet-}$ generation in the aorta, ${ }^{42}$ we surmise that eNOS is a likely source of $\mathrm{O}_{2}{ }^{\bullet-}$ production in the BAV aorta.

In conclusion, our data reveal an environment of heightened oxidative stress associated with impaired oxidative defense and increased lipid peroxidation in the aortic wall in the setting of BAV-associated aortopathy. The influence of oxidative stress on medial SMCs and on the medial matrix microarchitecture and biomechanical integrity in the aortic wall of patients with BAV is the focus of our ongoing work.

\section{Limitations of the Study}

A limitation to our study is the limited number of aortic specimens for certain patient subsets. Specifically, we were unable to further delineate the role of valvulopathy (aortic valve regurgitation and/or aortic valve stenosis) or BAV morphotype in the different parameters measured in our study. Therefore, severe aortic valve regurgitation and/or stenosis were potential confounding factors in our experiments. Despise these clinical limitations, we recently have demonstrated that aortic valve regurgitation influenced Sod gene expression in aneurysmal specimens isolated from patients with TAV, whereas aortic valve stenosis had no effect. $^{8}$

\section{Clinical Implications of the Study}

Despite the fact that BAV is the most common cardiac anomaly, occurring in $1 \%$ to $2 \%$ of the population, the cellular and molecular mechanisms underlying the associated aortopathy remain largely unknown. We propose a role for oxidative stress in the BAV-associated aortopathy, whereby $\mathrm{O}_{2}{ }^{--}$and possibly other ROS lead to oxidative damages in the aortic wall. Despite an increase in the peroxidase component of the antioxidant response, the specific antioxidant defense against $\mathrm{O}_{2}{ }^{\bullet-}$ (ie, superoxide dismutase) was not increased in aortic BAV specimens. Because oxidative stress is a well-known disruptor of ECM homeostasis, we suggest that oxidative stress is involved in the ECM derangements associated with BAV aortopathy. Understanding the exact link between oxidative stress and ECM in the context of BAV aortopathy may lead to the discovery of new therapeutic targets to prevent and/or delay aneurysm formation and/or rupture in patients with BAV. In addition, with the development of imaging methods allowing for in vivo detection of oxidative stress, ${ }^{45-47}$ our work may support the need to design new diagnostic tools that would utilize oxidative stress as a marker of BAV aortopathy.

\section{Conflict of Interest Statement}

Authors have nothing to disclose with regard to commercial support.

The authors gratefully acknowledge Deborah Cleary for technical assistant in patient specimen preparation, bioassay performance, and related analysis and Kristin Valchar and Julie Schreiber for assistance with institutional review board protocols and informed patient consent. We thank Forozan Navid for help with aortic specimen acquisition and Dr Andrew Althouse at the Heart and Vascular Institute for assistance with statistical analyses. 


\section{References}

1. Roberts WC. The congenitally bicuspid aortic valve. A study of 85 autopsy cases. Am J Cardiol. 1970;26:72-83.

2. Ward C. Clinical significance of the bicuspid aortic valve. Heart. 2000;83:81-5.

3. Ejiri J, Inoue N, Tsukube T, Munezane T, Hino Y, Kobayashi S, et al. Oxidative stress in the pathogenesis of thoracic aortic aneurysm: protective role of statin and angiotensin II type 1 receptor blocker. Cardiovasc Res. 2003;59:988-96.

4. Fiorillo C, Becatti M, Attanasio M, Lucarini L, Nassi N, Evangelisti L, et al. Evidence for oxidative stress in plasma of patients with Marfan syndrome. Int J Cardiol. 2010;145:544-6.

5. Xiong W, Mactaggart J, Knispel R, Worth J, Zhu Z, Li Y, et al. Inhibition of reactive oxygen species attenuates aneurysm formation in a murine model. Atherosclerosis. 2009;202:128-34.

6. Phillippi JA, Klyachko EA, Kenny JP, Eskay MA, Gorman RC, Gleason TG. Basal and oxidative stress-induced expression of metallothionein is decreased in ascending aortic aneurysms of bicuspid aortic valve patients. Circulation. 2009;119:2498-506.

7. Sievers HH, Schmidtke C. A classification system for the bicuspid aortic valve from 304 surgical specimens. J Thorac Cardiovasc Surg. 2007;133:1226-33.

8. Phillippi JA, Hill JC, Billaud M, Green BR, Kotlarczyk MP, Gleason TG. Bicuspid aortic valve morphotype correlates with regional antioxidant gene expression profiles in the proximal ascending aorta. Ann Thorac Surg. 2017;140:79-87.

9. Sievers HH, Stierle U, Mohamed SA, Hanke T, Richardt D, Schmidtke C, et al. Toward individualized management of the ascending aorta in bicuspid aortic valve surgery: the role of valve phenotype in 1362 patients. J Thorac Cardiovasc Surg. 2014;148:2072-80.

10. Zielonka J, Vasquez-Vivar J, Kalyanaraman B. Detection of 2-hydroxyethidium in cellular systems: a unique marker product of superoxide and hydroethidine. Nat Protoc. 2008;3:8-21.

11. Weydert CJ, Cullen JJ. Measurement of superoxide dismutase, catalase and glutathione peroxidase in cultured cells and tissue. Nat Protoc. 2010;5:51-66.

12. Griendling KK, Touyz RM, Zweier JL, Dikalov S, Chilian W, Chen YR, et al. Measurement of reactive oxygen species, reactive nitrogen species, and redoxdependent signaling in the cardiovascular system: a scientific statement From the American Heart Association. Circ Res. 2016;119:e39-75.

13. Hoaglin DC, Iglewicz B. Fine-tuning some resistant rules for outlier labeling. $J$ Am Stat Assoc. 1987;82:1147-9.

14. Wu D, Shen YH, Russell L, Coselli JS, LeMaire SA. Molecular mechanisms of thoracic aortic dissection. J Surg Res. 2013;184:907-24.

15. Gleason TG. Heritable disorders predisposing to aortic dissection. Semin Thorac Cardiovasc Surg. 2005; 17:274-81.

16. Elefteriades JA, Farkas EA. Thoracic aortic aneurysm clinically pertinent controversies and uncertainties. J Am Coll Cardiol. 2010;55:841-57.

17. Olsson C, Thelin S, Stahle E, Ekbom A, Granath F. Thoracic aortic aneurysm and dissection: increasing prevalence and improved outcomes reported in a nationwide population-based study of more than 14,000 cases from 1987 to 2002. Circulation. 2006;114:2611-8.

18. Michelena HI, Khanna AD, Mahoney D, Margaryan E, Topilsky Y, Suri RM, et al. Incidence of aortic complications in patients with bicuspid aortic valves. JAMA. 2011;306:1104-12.

19. Sabet HY, Edwards WD, Tazelaar HD, Daly RC. Congenitally bicuspid aortic valves: a surgical pathology study of 542 cases (1991 through 1996) and a literature review of 2,715 additional cases. Mayo Clin Proc. 1999;74:14-26.

20. Michelena HI, Desjardins VA, Avierinos JF, Russo A, Nkomo VT, Sundt TM, et al. Natural history of asymptomatic patients with normally functioning or minimally dysfunctional bicuspid aortic valve in the community. Circulation. 2008; 117:2776-84.

21. Tzemos N, Therrien J, Yip J, Thanassoulis G, Tremblay S, Jamorski MT, et al. Outcomes in adults with bicuspid aortic valves. JAMA. 2008;300:1317-25.

22. Keane MG, Wiegers SE, Plappert T, Pochettino A, Bavaria JE, Sutton MG. Bicuspid aortic valves are associated with aortic dilatation out of proportion to coexistent valvular lesions. Circulation. 2000;102(19 suppl 3):III35-9.

23. Tsamis A, Phillippi JA, Koch RG, Krawiec JT, D’Amore A, Watkins SC, et al. Extracellular matrix fiber microarchitecture is region-specific in bicuspid aortic valve-associated ascending aortic aneurysms. J Thorac Cardiovasc Surg. 2016; 151:1718-28.e5

24. Phillippi JA, Green BR, Eskay MA, Kotlarczyk MP, Hill MR, Robertson AM, et al. Mechanism of aortic medial matrix remodeling is distinct in patients with bicuspid aortic valve. J Thorac Cardiovasc Surg. 2014;147:1056-64.

25. Tsamis A, Phillippi JA, Koch RG, Pasta S, D'Amore A, Watkins SC, et al. Fiber micro-architecture in the longitudinal-radial and circumferential-radial planes of ascending thoracic aortic aneurysm media. J Biomech. 2013;46:2787-94.
26. Pichamuthu JE, Phillippi JA, Cleary DA, Chew DW, Hempel J, Vorp DA, et al. Differential tensile strength and collagen composition in ascending aortic aneurysms by aortic valve phenotype. Ann Thorac Surg. 2013;96:2147-54.

27. Pasta S, Phillippi JA, Gleason TG, Vorp DA. Effect of aneurysm on the mechanical dissection properties of the human ascending thoracic aorta. J Thorac Cardiovasc Surg. 2012;143:460-7.

28. Phillippi JA, Eskay MA, Kubala AA, Pitt BR, Gleason TG. Altered oxidative stress responses and increased type I collagen expression in bicuspid aortic valve patients. Ann Thorac Surg. 2010;90:1893-8.

29. Pereira L, Lee SY, Gayraud B, Andrikopoulos K, Shapiro SD, Bunton T, et al. Pathogenetic sequence for aneurysm revealed in mice underexpressing fibrillin-1. Proc Natl Acad Sci U S A. 1999;96:3819-23.

30. Yang HH, van Breemen C, Chung AW. Vasomotor dysfunction in the thoracic aorta of Marfan syndrome is associated with accumulation of oxidative stress. Vasc Pharmacol. 2010;52:37-45.

31. Steed MM, Tyagi N, Sen U, Schuschke DA, Joshua IG, Tyagi SC. Functional consequences of the collagen/elastin switch in vascular remodeling in hyperhomocysteinemic wild-type, eNOS-/-, and iNOS-/- mice. Am J Physiol. 2010;299: L301-11.

32. Folkersen L, Wagsater D, Paloschi V, Jackson V, Petrini J, Kurtovic S, et al. Unraveling divergent gene expression profiles in bicuspid and tricuspid aortic valve patients with thoracic aortic dilatation: the ASAP study. Mol Med. 2011;17: 1365-73.

33. Arcucci A, Ruocco MR, Albano F, Granato G, Romano V, Corso G, et al. Analysis of extracellular superoxide dismutase and Akt in ascending aortic aneurysm with tricuspid or bicuspid aortic valve. Eur J Histochem. 2014;58:2383.

34. Branchetti E, Poggio P, Sainger R, Shang E, Grau JB, Jackson BM, et al. Oxidative stress modulates vascular smooth muscle cell phenotype via CTGF in thoracic aortic aneurysm. Cardiovasc Res. 2013;100:316-24.

35. Hill BG, Haberzettl P, Ahmed Y, Srivastava S, Bhatnagar A. Unsaturated lipid peroxidation-derived aldehydes activate autophagy in vascular smooth-muscle cells. Biochem J. 2008;410:525-34.

36. Cabre A, Girona J, Vallve JC, Heras M, Masana L. Cytotoxic effects of the lipid peroxidation product 2,4-decadienal in vascular smooth muscle cells. Atherosclerosis. 2003;169:245-50.

37. Holvoet P, Collen D. Oxidation of low density lipoproteins in the pathogenesis of atherosclerosis. Atherosclerosis. 1998;137(suppl):S33-8.

38. Holvoet P, Mertens A, Verhamme P, Bogaerts K, Beyens G, Verhaeghe R, et al. Circulating oxidized LDL is a useful marker for identifying patients with coronary artery disease. Arterioscler Thromb Vasc Biol. 2001;21:844-8.

39. Shimoni S, Bar I, Zilberman L, George J. Autoantibodies to oxidized low-density lipoprotein in patients with aortic regurgitation: association with aortic diameter size. Cardiology. 2014;128:54-61.

40. Fruhwirth GO, Moumtzi A, Loidl A, Ingolic E, Hermetter A. The oxidized phospholipids POVPC and PGPC inhibit growth and induce apoptosis in vascular smooth muscle cells. Biochim Biophys Acta. 2006;1761:1060-9.

41. Ejiri J, Inoue N, Tsukube T, Munezane T, Hino Y, Hunter GC. Oxidative stress in human abdominal aortic aneurysmal and occlusive disease. Cardiovasc Res. 1999;59:39-45.

42. Kotlarczyk MP, Billaud M, Green BR, Hill JC, Shiva S, Kelley EE, et al. Regional disruptions in endothelial nitric oxide pathway associated with bicuspid aortic valve. Ann Thorac Surg. 2016;102:1274-81.

43. Mohamed SA, Radtke A, Saraei R, Bullerdiek J, Sorani H, Nimzyk R, et al. Locally different endothelial nitric oxide synthase protein levels in ascending aortic aneurysms of bicuspid and tricuspid aortic valve. Cardiol Res Pract. 2012;2012:165957.

44. Pisano C, Maresi E, Balistreri CR, Candore G, Merlo D, Fattouch K, et al. Histological and genetic studies in patients with bicuspid aortic valve and ascending aorta complications. Interact Cardiovasc Thorac Surg. 2012;14:300-6.

45. Prunty MC, Aung MH, Hanif AM, Allen RS, Chrenek MA, Boatright JH, et al. In vivo imaging of retinal oxidative stress using a reactive oxygen speciesactivated fluorescent probe. Invest Ophthalmol Vis Sci. 2015;56:5862-70.

46. Tanase M, Urbanska AM, Zolla V, Clement CC, Huang L, Morozova K, et al. Role of carbonyl modifications on aging-associated protein aggregation. Sci Rep. 2016;6:19311.

47. Khoo NK, Cantu-Medellin N, St Croix C, Kelley EE. In vivo immuno-spin trapping: imaging the footprints of oxidative Stress. Curr Protoc Cytom. 2015;74: 12.42.1-11.

Key Words: aortic aneurysm, oxidative stress, vascular biology, bicuspid aortic valve 